\title{
NINCAPANG KAWAGEDAN NGWACEN AKSARA BALI SISIA KELAS IVA SD NEGERI 2 SAKTI NUSA PENIDA WARSA 2018/2019 ANTUK MEDIA TOPI AKSARA SANE KASURAT NGANGGEN APLIKASI BALINESE FONT AND KEYBOARD
}

\author{
Ni Kdk Sintha Cahyani ${ }^{1}$, I.B. Md Ludy Paryatna ${ }^{1}$, I Kt Paramarta ${ }^{2}$ \\ Program Studi Pendidikan Bahasa Bali \\ Universitas Pendidikan Ganesha \\ Singaraja, Indonesia \\ e-mail: \{sinthaacahyanii@yahoo.co.id, ludy.paryatna, \\ ketut.paramarta\}@undiksha.ac.id
}

\begin{abstract}
KUUB
Tetilikan puniki matetujon nlatarang indik (1) tata cara media pembelajaran topi aksara sane kasurat antuk aplikasi Balinese font and keyboard (2) kawentenan media pembelajaran topi aksara sane kasurat antuk aplikasi Balinese font and keyboard nincapang kawagedan ngwacen aksara Bali sisia kelas IVA SD Negeri 2 Sakti Nusa Penida (3) penampen sisia indik media pembelajaran topi aksara sane kasurat antuk aplikasi Balinese font and keyboard. Ring tetilikan puniki kaanggen aplikasi Balinese font and keyboard mawinan kawentenan aplikasinyane sane durung wenten kaanggen ring tetilikan sane lianan lan wentuk aksaranyane sane pinih becik yening kasaihang sareng aplikasi aksara Bali sane lianan sane akehan aksaranyane matumpuk-tumpuk, sekadi imba silih tunggil kawentenan pepet lan cecek yening ring aplikasi lianan matumpuk, nanging ring aplikasi Balinese font and keyboard nenten matumpuk. Jejering tetliknyane inggih punika guru basa Bali lan sisia kelas IVA SD Negeri 2 Sakti Nusa Penida lan panandang tetiliknyane inggih punika ngawigunayang media pembelajaran topi aksara sane kasurat antuk aplikasi Balinese font and keyboard lan kawagedan sisia ngwacen aksara Bali sane becik. Ring tetilik puniki nganggen kalih deskriptif, minakadi deskriptif kualitatif lan deskriptif kuantitatif. Tetilik puniki nganggen tigang piranti ritatkala mupulang data minakadi pratiyaksa, tes lan angket/kuesioner. Pikolih ring tetilik puniki inggih punika : (1) tata cara pamargin tetilik kamargiang saking pangawit sekadi panganjali, ring inti sekadi ngicen materi lan nampilang media topi aksara miwah pamuput sekadi nyutetang paplajahan ngantos parama Santi. (2) pikolih tes kawagedan ngwacen aksara Bali sisia kabaos nincap minakadi siklus I pikolih rerata 72,53 lan siklus II 80,13. (3) pikolih rerata panampen sisia indik media pembelajaran topi aksara akehnyane 27,67.
\end{abstract}

Kruna jejaton : aksara, aplikasi, media, ngwacen 


\section{ABSTRACT}

This study aims to explain about (1) the procedure for literary hat media written with the application of Balinese font and keyboard (2) the existence of literary hat learning media written with the application Balinese font and keyboard can increase the knowledge of reading Balinese characters IVA grade 2 Sakti Elementary School Nusa Penida (3) the opinions of students about the literary hat learning media written with the Balinese font and keyboard application. In this study, the Balinese font and keyboard application was used because the form of the script was neater compared to other applications. In addition, with this application, there is a new touch in the world of education. The subject of this study is the Balinese language teacher and class IVA 2 Sakti Nusa Penida Elementary School and the object is the use of literary hat media written with the Balinese font and keyboard application and students' knowledge in reading Balinese script. This study used two descriptive methods, qualitative descriptive and quantitative descriptive methods. This study used 3 tools or materials, namely observation, tests, and questionnaires. The result of this study are: (1) the procedure for conducting this research from the beginning, which is giving panganjali, giving material in the core of activity and using literary hat media, to the last one when concluding the lesson and giving parama Santi. (2) The results of this study are said to increase, as evidenced by the average Cycle I which is 72.53 and cycle II 80.13. (3) The average results of students' opinions regarding literary hat learning are 27.67 .

Key words: aksara, application, media, reading

\section{PURWAKA}

Pelajaran sane pinih becik patut kapolihang olih para sisia ring aab jagat sekadi mangkin. Punika makasami nenten wantah prasida kapolihang ring pakuub kluarga lan krama banjar kemanten, nanging sane kautamayang inggih punika pendidikan formal. Tatujon pendidikan inggih punika nincapang kaweruhan bangsa lan nincapang jadma Indonesia sane sapatutnyane, inggih punika jadma sane religius utawi manut tekening agama lan satya tekening Ida Sang Hyang Widhi Wasa madue kaweruhan, kauripan, lan parilaksana sane becik.

Sajeroning plajahan basa Bali wenten petang soroh kawagedan sane patut kaplajahin antuk para sisia, minakadi kawagedan miarsayang (keterampilan menyimak), kawagedan mababaosan (keterampilan berbicara), kawagedan ngawacen (keterampilan membaca), miwah kawagedan nyurat (keterampilan menulis) (Suwija,2014:9). Silih sinunggih kawagedan sane patut katelebin inggih punika kawagedan ngwacen. Kawagedan ngwacen wenten kakalih, inggih punika kawagedan ngwacen aksara Bali lan latin. Sisia kaaptiang prasida lan waged ngwacen aksara Bali, duaning ngwacen aksara Bali mabuat pesan ring sajeroning plajahan basa Bali (Suwija,2014:11).

Aksara inggih punika ceciren utawi pralambang basa sane kawetuang olih imanusa. Aksara Bali punika aksara sane tradisional wenten ring Bali. Yening para sisia nanten prasida ngwacen aksara Bali sane becik, sinah ngawinang sisia punika molihang pikobet ngwacen ring undagan sekolah selanturnyane. Panilik taler nyingakin kantun makehan guru basa Bali nenten nganggen media paplajahan sane patut ri kala ngemargiang plajahan ring kelas. Guru sepatutnyane nganggen media sane menarik mangda sisia sayan seneng ri kala nyarengin paplajahan. Media paplajahan menarik inggih punika paplajahan sane karincikang olih guru sumangdane media paplajahan punika mabinayan ring paplajahan sane sampun sering kamargiang.

Media paplajahan sane menarik ring tetilik puniki panilik nganggen serana topi aksara sane kasurat antuk Balinese font and keyboard. Media topi aksara puniki kasurat nganggen aplikasi Balinese Font and Keyboard sane wenten ring IOS. Sadurung nyurat aksara, guru ngrereh gambar utawi foto samian sisia kelas IVA SD Negeri 2 Sakti Nusa Penida, usan punika ring gambar punika kadagingin topi tur kasuratin kruna sane nganggen aksara Bali . Tata cara ngamargiang media paplajahan puniki inggih punika guru nyiyagayang nomor 1-30 ring LCD, usan punika sisia magilir milih siki nomor tur ngwacen sasuratan sane wenten ring topi aksara sane kasurat antuk Balinese font and keyboard punika. Media topi aksara sane kasurat antuk Balinese font and keyboard puniki madue kaluwihan inggih punika (1) guru praktis 
ngaryanin media puniki santukan sampun wenten ring HP khususnyane sane nganggen Apple, sekadi Iphone miwah Ipad, (2) nenten perlu mesuang jinah akeh anggen numbas kertas, spidol, miwah sane sarana sane lianan santukan sampun prasida kasurat nganggen aplikasi, (3) prasida kakaryanin ring genah dija kemanten, (4) nenten akeh nelasang tenaga utawi bayu ri kala ngaryanin media puniki. Dadosnyane media paplajahan topi aksara sane kasurat antuk Balinese font and keyboard puniki prasida ngawinang sisia sayan meled tur seneng ngamiletin paplajahan ngwacen aksara Bali. Yening sisia sampun seneng, sinah sisia sayan dangan ngresepang napi sane sampun kaplajahin Manut kadi dadalan pikobet ring ajeng, bantang pikobet ring tetilikan puniki inggih puniki, minakadi : 1. Sapunapi tata cara ngawigunayang media pembelajaran topi aksara sane kasurat antuk aplikasi balinese font and keyboard ring paplajahan ngwacen aksara Bali majeng sisia kelas IVA SD Negeri 2 Sakti Nusa Penida? 2. Sapunapi pangresep sisia kelas IVA SD Negeri 2 Sakti Nusa Penida sawusan ngawigunayang media pembelajaran topi aksara sane kasurat antuk aplikasi balinese font and keyboard ring paplajahan ngwacen aksara Bali? 3. Sapunapi penampen sisia kelas IVA SD Negeri 2 Sakti Nusa Penida ring kawentenan media topi aksara sane kasurat antuk Balinese font and keyboard ring paplajahan ngwacen aksara Bali?

Manut kadi bantang pikobet sane kaunggahang ring ajeng, tatujon tetilik puniki inggih punika, minakadi : 1. Mangda uning tata cara ngawigunayang media pembelajaran topi aksara sane kasurat antuk aplikasi balinese font and keyboard ring paplajahan ngwacen aksara Bali majeng ring sisia kelas IVA SD Negeri 2 Sakti Nusa Penida. 2. Mangda uning pangresep sisia kelas IVA SD Negeri 2 Sakti Nusa Penida sawusan ngawigunayang media topi aksara sane kasurat antuk Balinese font and keyboard ring paplajahan ngwacen aksara Bali. 3 . Mangda uning panampen sisia kelas IVA SD Negeri 2 Sakti Nusa Penida indik kawentenan media topi aksara sane kasurat antuk Balinese font and keyboard ring paplajahan ngwacen aksara Bali.

Ring sajeroning tetilik puniki wenten kawigunannyane, minakadi kawigunan pamucuk, pikolih tetilik puniki kaaptiang prasida ngicen kawigunan ring sapanglimbak serana paplajahan anggen nincapang kawagedan ngwacen aksara tur prasida ngranayang sisinayane sayan aktif ring sajeroning pamargi pangajah-ajahan. Taler pikolih tetilikan puniki kaaptiang prasida anggen sapanglimbak widya pengajah-ajahan, cutetnyane papaljahan basa
Bali. Wenten taler kawigunan panglimbak sumangdene mawiguna majeng ring sisia prasida kadadosang pangalaman, majeng ring guru mangda prasida nglimbakang ri sajeroning paplajahan, majeng ring sekolah mangda prasida kaanggen tetimbangan ri tatkala ngaryanin penilaian, miwah majeng ring panilik sane selanturnyane mangda prasida ngicenin rerawatan.

Manut Zuriah (2009: 6), teori punika silih sinunggil utsaha sane kanggen panyangkaan ritatkala jagi nlatarang pikobet sane pacang kamargiang. Ri kala nglaksanayang tetilikan patut kadasarin antuk kaweruhan sane sampun kacumpuin (Arikunto, 2005: 58). Kaweruhan sane sampun wenten malih kaselehin mangda kauiningin sane sampun patut lan sane nenten patut kanggen ring tetilik. Sepat siku-siku sane kanggen ring tetilik puniki inggih punika (1) Ngwacen Aksara Bali, (2) Media Topi Aksara, (3) Balinese Font and Keyboard.

\section{KRAMANING TETILIK}

Kramaning tetilik inggih punika tata cara sane kalaksanayang ring kawentenan penelitian. Tata cara puniki ngranjing ring laksana utama sane kalaksanayang olih peneliti sane kaange nyawis pikobet sane wenten (Wendra, 2014 : 31). Manut sane sampun katelatarang tata cara sane kalaksanayang inggih punika 1) Palihan Tetilikan (Rencana Penelitian), 2) Jejering lan Penandang Tetilik (Subjek dan Objek Penelitian), 3) Panuntut Tetilikan (Prosedur Pelaksanaan), 4) Pangrencana (Perencanaan), 5) Panglaksana (Pelaksanaan), 6) Seseleh lan Pamastika (Observasi/Evaluasi), 7) Mulat Wali (Refleksi), 8) Piranti (Instrumen) lan Pidabdab Mupulang Data , lan 9) Tatacara Data Tureksa lan Sepat Sida.

Palihan tetilik inggih punika tatacara sane kanggen ngatur latar (setting) tetilik sumangdane panilik ngamolihang data sane patut (valid) manut tekening karakteristik variable lan tetujon tetilik (Sutama, 2009:15). Manut baos sujana punika prasida panilik cutetang indik palihan tetilik inggih punika pidabdab sane kanggen ri kala ngalaksanayang tetilik mangda pikobet sane katilikin nganggen pidabdab sane patut. Ring tetilik sane kalaksanayang puniki, sampun janten tetilik puniki nganggen PTK (Penelitian Tindakan Kelas). Kabaos Penelitian Tindakan Kelas duaning tetilikan puniki kalaksanayang ring kelas antuk makudang tata cara sane kanggen muputang pikobet sane kapolihang guru ri kala ngamargiang plajahan (Wendra, 2011:45). Palihan tetilik tindakan kelas puniki kanggen santukan anut sareng tetujon saking tetilik puniki, inggih punika mautsaha mecikang lan 
nincapang pikolih malajah lan ngajahin sane kalaksanayang ring kelas. Akeh siklus sane kalaksanayang sajeroning tetilik tindakan kelas dahat mapaiketan sareng sepat sida. Cutetnyane, yening sepat sida punika prasida kapolihang ring kalih siklus, raris tetilik prasida kapuputang.

Jejering mabuat pesan ring sajeroning tetilik, jejering taler kabaos mautama ring tetilik duaning saking jejering punika prasida kapolihang data sane kakaryaninang tur katilikin olih panilik. Tetilik puniki kalaksanayang ring SD Negeri 2 Sakti Nusa Penida, wenten taler populasi sane kanggen ring tetilik punika inggih punika sisia kelas IVA SD Negeri 2 Sakti Nusa Penida, nanging jejering sane kanggen inggih punika makasami sisia lan guru basa Bali kelas IVA SD Negeri 2 Sakti Nusa Penida warsa 2019 sane akehnyane 15 sisia. Sisia lanang 7 diri miwah sisia istri 8 diri. Ring tetilik puniki nilikin indik kawagedan sisia ngwacen aksara Bali ring kelas IVA. Panilik ngawentenan tetilik puniki ring sisia kelas IVA, duaning ring kelas puniki kerasayang kantun makeh sisia sane kirang waged ngwacen aksara Bali, tur makeh sisia sane nenten urati ri kala polih plajahan basa Bali. Kawentenane puniki prasida kacingakin saking nilai sisia sane kantun kirang ri kala ngwacen aksara Bali. Kantun makeh sisia sane durung prasida ngamolihang nilai nganutin $\mathrm{KKM}$. KKM sane kanggen ring kelas IVA SD Negeri 2 Sakti Nusa Penida inggih punika 75.

Penandang tetilik inggih punika parindikan sane pacang katureksain ring sajeroning tetilikan. Penandang sane marupa proses inggih punika ngawigunayang media paplajahan masarana topi aksara sane kasurat antuk Balinese font and keyboard. Penandang sane marupa produk inggih punika kawagedan sisia ngwacen aksara Bali cutetnyane aksara wianjana, pengangge aksara miwah angka.

Tetilik puniki nganggen tetilik tindakan kelas, mawinan asapunika tetilikan puniki jagi kalaksanayang antuk makudang-kudang siklus. Akehnyane siklus sane kanggen ring tetilik puniki nganutin pikolih sane kapolihang, yening pikolih sane kapolihang kirang panilik jagi ngawawanin malih ngantos pikolih sane kapolihang becik. Ring sajeroning siklus punika, wenten makudang-kudang geginan sane patut kalaksanayang minakadi mulat wali awal, pangerencana tetilik, palaksanan tindakan, pamastika (evaluasi) lan mulat wali.

Pangerencana ring sajeroning siklus kasusun anggen mecikang peplajahan (Sanjaya, 2012:78). Ring pangrencana puniki panilik pacang nyayagaang piranti-piranti sane pacang kanggen ri kala ngawentenang tetilik puniki. Wenten makudang geginan sane pacang kalaksanayang inggih punika sekadi: (1) Panilik sareng guru nyelehin silabus sane kanggen ring paplajahan mangda tatujon paplajahan prasida mamargi. (2) Panilik lan guru, sareng-sareng makarya RPP anggen piranti nglaksanayang paplajahan. (3) Panilik nlatarang majeng ring guru mata pelajaran basa Bali ring kelas IVA SD Negeri 2 Sakti Nusa Penida ngenenin indik tata cara ngamargiang paplajahan nganggen media topi aksara sane kasurat antuk Balinese font and keyboard ring IOS sane jagi kamargiang. (4) Panilik sareng guru makarya piranti tetilik. Wenten piranti tetilik sane kanggen inggih punika panuntun pratiyaksa, kuisioner/angket, lan test. Sasampun ngawentenang makudangkudang palihan pangrencana tetilik, selanturnyane kamargiang sekancan pangrencana sane sampun kakaryanin. Tetilik puniki pacang kalaksanayang kalih siklus.

Kawentenan seseleh matetujon nguningayang pekaryan sane kalaksanayang olih sisia ri kala paplajahan puniki kalaksanayang. Seseleh puniki kalaksanayang ring sajeroning geginan malajah kalaksanayang, ngawit ngawitin paplajahan ngantos puput paplajahane. Tetujon nglaksanayang seseleh mangda uning kekirangan ring sajeroning panglaksana sane kalaksanayang. Seseleh kalaksanayang nganggen panuntun pratiyaksa sane sampun kakaryanin olih panilik nganutin tuntunan sareng dosen. Saking pikolih sane kapolihang ring seseleh sane sampun kalaksanayang punika, prasida kacingakin sapunapin pikolih media topi aksara sane kasurat antuk Balinese font and keyboard ring IOS ring sajeroning kawagedan sisia ngwacen aksara Bali. Pemastika inggih punika tatacara nguningin panglimbak pikolih mlajah sisia ring pengajahan lan nguningin sapunapi pikolih malajah sane kapolihang, napike anut utawi nenten sareng tatujon paplajahan. Usan punika kalaksanayang Mulat wali ungkur paplajahan, utawi sesampun materi plajahan punika usan katelatarang. Geginan puniki kalaksanayang pinaka tata cara nureksain pikolih sane kapolihang sausan kalaksanayang media topi aksara sane kasurat antuk Balinese font and keyboard. Napike pikolihnyane becik utawi nenten.

Wenten makudang-kudang pidabdab mupulang data lan pirantinyane (instrumen), minakadi : 1) Pidabdab praktiasa sane kanggen mupulang data ngeninin indik gaginan sisia lan guru ri kala kawentenan plajahan, 2) Pidabdab angket kasaratang ring sajeroning mupulang data indik panampen sisia majeng ring pamargin plajahan, lan 3) Kramaning tes inggih punika serana sane kanggen nyelehin kaweruhan sisia ngenenin indik akademik lan 
kawagedan pinaka pikolih sisia ring paplajahan. Manut KKM SD Negeri 2 Sakti Nusa Penida antuk palajahan Basa Bali inggih punika 75 . Dadosnyane sisia sampun kabaos tuntas yening sampun ngamolihang nilai pinih akidik 75 .

Data sane ngenenin indik pangresep sisia ring paplajahan ngwacen aksara Bali katilikin nganggen teknik deskriptif kuantitatif. Penilaian pangresep sisia kelas IVA SD Negeri 2 Sakti Nusa Penida kalaksanayang nganggen Penilaian Acuan Patokan (PAP). Panampen sisia pacang katilikin nganggen teknis analisis deskripsi kuantitatif lan kualitatif. Tetilikan punika kabaosang sampun mikolihang (berhasi) yening $60 \%$ sisia ngicen panampen sane positif. Palet sane kalaksanayang sajeroning nilikin data panampen sisia inggih punika: (1) Ngrereh Skor Maksimal ldeal (SMI) saking kuesioner sane kaicen. SMI karereh antuk tata cara ngitung makeh item sane kaicen lan bobot saking soang-soang item. (2) Ngrereh rata-rata ideal (Mi) antuk kuesioner punika ngangge rumus:

Mi $=\frac{1}{2}$ (skor maksimal ideal + skor minimal ideal)

(3) Ngrereh Standar Deviasi Ideal (SDi) antuk kuesioer punika ngangge rumus:

Sdi $=\frac{1}{3} \times \mathrm{Mi}$

\section{PIKOLIH LAN TETEPASAN}

Tetilik puniki kalaksanayang ring kelas IVA SD Negeri 2 Sakti Nusa Penida, genahnyane ring Dusun Pundukaha Kelod, Desa Bunga Mekar, Kecamatan Nusa Penida, Kabupaten Klungkung. Akeh sisia sane wenten ring kelas IVA puniki wenten 15 diri, 7 sisia lanang miwah 8 sisia istri. Sane pinaka guru basa Bali ring kelas puniki mawasta I Kadek Suardana, S.Pd. SD sane kaajak penilik sareng-sareng ngwantu tetilikan puniki. Tetilik puniki kalaksanayang ring bulan April ngantos Mei 2019 sane madaging indik pangrencana lan pelaksana tindakan. Paplajahan Basa Bali ring kelas IVA puniki kalaksanayang wantah apisan ring awuku inggih punika ring rahina Sukra galah 6 - 7 (11.3012.30).

Ring pinanggal 19 April 2019, penilik ngalaksanayang praktiaksa nyingakin pamargin paplajahan sane kalaksanayang olih guru. Pangawit guru ngaturang panganjali, ngabsen, lan nagingin jurnal. Usan punika kalanturang guru ngicen materi sane kasobiahang wantah nganggen metode ceramah lan nenten nganggen media. Sisia ring kelas IVA sami nyatet lan nenten wenten sane mataken santukan gurunyane galak.

\begin{tabular}{|c|c|c|}
\hline No. & Rahina/Pinanggal & Pelaksana \\
\hline 1 & $\begin{array}{c}\text { Rabu, } 17 \text { April } \\
2019\end{array}$ & $\begin{array}{l}\text { Observasi } \\
\text { Sekolah }\end{array}$ \\
\hline 2 & $\begin{array}{c}\text { Sukra, } 19 \text { April } \\
2019\end{array}$ & $\begin{array}{c}\text { Observasi Guru } \\
\text { ring Kelas }\end{array}$ \\
\hline 3 & $\begin{array}{c}\text { Sukra, } 26 \text { April } \\
2019\end{array}$ & $\begin{array}{l}\text { Patemon I } \\
\text { (siklus I) }\end{array}$ \\
\hline 4 & Sukra, 3 Mei 2019 & $\begin{array}{l}\text { Patemon II } \\
\text { (siklus I) }\end{array}$ \\
\hline 5 & $\begin{array}{c}\text { Sukra, } 10 \text { Mei } \\
2019\end{array}$ & $\begin{array}{l}\text { Patemon I } \\
\text { (siklus II) }\end{array}$ \\
\hline 6 & $\begin{array}{c}\text { Sukra, } 17 \text { Mei } \\
2019\end{array}$ & $\begin{array}{l}\text { Patemon II } \\
\text { (siklus II) }\end{array}$ \\
\hline 7 & $\begin{array}{c}\text { Sukra, } 17 \text { Mei } \\
2019\end{array}$ & $\begin{array}{l}\text { Nagingin } \\
\text { Kuesioner } \\
\text { risampun } \\
\text { kalaksanayang } \\
\text { Tindakan }\end{array}$ \\
\hline
\end{tabular}

Pikolih kapertama sadurung nganggen media topi aksara sane kasurat antuk aplikasi Balinese font and keyboard inggih punika 65,13. Riantukan pikolih ngwacen aksara Bali kari kirang, sarat kalaksanayang paobahan ritatkala ngamargiang paplajahan ngwacen aksara Bali nganggen foto sisia tur kaedit nganggen topi sane madaging sasuratan aksara Bali sane kasurat antuk aplikasi Balinese font and keyboard anggen ngamijilang minat malajah sisia kelas IVA SD Negeri 2 Sakti.

Pikoloh tetilik kaping kalih inggih punika indik pikolih siklus I. Panglaksana ring siklus I puniki kalaksanayang ring Sukra (26 April lan 3 Mei 2019) jam ke $6-7$. Wenten makudangkudang sarana sane kaanggen penilik, minakadi: a) Nyiagayang materi sane mapaiketan sareng ngwacen aksara Bali. b) Nyiagayang media paplajahan marupa sasuratan kruna lan lengkara sane kaanggen ritatkala ngalaksanayang paplajahan inggih punika media topi aksara sane kasurat nganggen Balinese font and keyboard. c) Nyiagayang piranti sane kaanggen ring lelintihan pangajah-ajah minakadi laptop, $L C D$, lan emoticon sane prasida katempel sane madue wentuk star, jempol, miwah pin penghargaan.

Pikolih rerata sane kapolihang ring siklus I puniki akehnyane 72,53. Lan pikolih persentase sane kapolihang sane anut sareng KKM wantah $33,33 \%$ (5 diri sane prasida ngamolihang nilai 75). Yening kacingak, makasamian tetilikan kabaos tuntas yening anut sareng KKM akeh 
nyane $75 \%$ lan prasida ngamolihang nilai 75 . Nika mawinan kalaksanayang siklus II.

Pikolih tetilikan kaping tiga inggih punika indik pikolih siklus II. Pangrencana ring tetilikan puniki kalaksanayang manut ring pikolih evaluasi lan mulat wali ring sikulus I. Pangrancana ring siklus II puniki mesib sareng pangrencana ring siklus I. Sane minayang inggih punika, kawentenan media foto sisia sane nganggen topi aksara sampun kalebihin.

Pikolih rerata sane kapolihang ring siklus II puniki akehnyane 80,13 . Lan pikolih persentase sane kapolihang sane anut sareng KKM punika sampun langkung, akeh nyane $86,67 \%$ (13 diri sane prasida ngamolihang nilai 75 miwah langkung). Pikolih punika nincap nyening kasaihang sareng pikolih tes ring siklus I, sane akehnyane $33,33 \%$ (5 diri sane prasida ngamolihang nilai 75 miwah langkung).

Pikolih sane kaping untat inggih punika indik panampen sisia ngawigunayang media topi aksara sane kasurat antuk aplikasi Balinese font and keyboard. Media paplajahan topi aksara sane kasurat antuk aplikasi Balinese font and keyboard anggen nincapang kawagedan ngwacen aksara Bali punika madue pangargan sane becik. Pikolih rerata sane kapolihang akehnyane 27,67 . Ring pikolih punika wantah 2 sisia sane madue pangargan positif $(13,33 \%)$ lan 13 sisia sane madue pangargan positif pisan $(86,67 \%)$. Lan prasida kacingak akehan sisia sane cumpu pisan sareng paplajahan ngwacen aksara Bali nganggen media topi aksara sane kasurat antuk aplikasi Balinese font and keyboard.

Manut ring pikolih awal indik pamargin paplajahan sane nenten kawantu sareng media paplajahan, punika ngaranayang pikolih sisia indik ngwacen aksara Bali akeh sane nenten anut sareng KKM. Riantukan punika anggen natasin pikobet kirang nyane pikolih kawagedan sisia ngwacen aksara Bali punika patut kalaksanayang media topi aksara sane kasurat nganggen apikasi Balinese font and keyboard. Media paplajahan topi aksara kaaptiang prasida nincapang keberhasilan sisia, ngamijilang motivasi malajah sisia, nincapang daya kreasi, ngawigunayang materi paplajahan punika nenten meweh kaengsapang lan prasida ngawiang panglaksana paplajahan punika trepti.

Tetepasan puniki nelatarang indik pikolihpikolih sane sanget mabuat ring tetilikan. Pikolih ring tetilikan puniki minakadi sane kapertama indik pamargin media paplajahan topi aksara kaanggen nincapang kawagedan ngwacen aksara Bali. Kaping kalih indik pikolih media paplajahan topi aksara kaanggen nincapang kawagedan ngwacen aksara Bali, lan kaping tiga indik penampen sisia ritatkala kamargiang media paplajahan topi aksara kaanggen nincapang kawagedan ngwacen aksara Bali. Makasamian punika jagi katelatarang ring sor puniki.

Pikolih sane kapartama indik tata cara utawi pamargin paplajahan nganggen media paplajahan topi aksara kaanggen nincapang kawagedan ngwacen aksara Bali sisia kelas IVA SD Negeri 2 Sakti Nusa Penida. Tata cara ngamargiang paplajahan nganggen media paplajahan topi aksara puniki kalaksanayang manut sareng pangrencana paplajahan sane sampun karincikan ring RPP. Risampune kalaksanayang mulatwali ring siklus I punika prasida kabaos sampun becik, nanging wenten kirangnyane. Kekirangan punika selanturnyane karerehang solusi mangda prasida ngamecikang pamargin paplajahan ring siklus II, solusinyane minakadi : 1) guru lan sisia ri tatkala nyarengin paplajahan punika patut kaimbangin sareng tanya jawab mangda suasana ring kelas punika idup. 2) ritatkala ngamargiang materi paplajahan punika nenten becik yening ceramah kemanten nanging teler perlu kaimbangin nganggen power point, gambar miwah sane lianan mangda sisia punika tertarik nyarengin paplajahan, 3) sisia punika kanikain nyatet indik materi sane sampun katelatarang, 4) sisia kaajak sareng-sareng nureksain pakaryan sawitra sane lianan mangda uning kaiwangannyane, 5) sadurung muputang paplajahan punika, sisia kaajak sareng-sareng nyutetang paplajahan.

Manut ring tata cara pamargin paplajahan, lelintihan pangajah-ajah punika sampun prasida mamargi becik lan efektif. Sisia uning indik pabinayan aksara pa sareng sa, lan prasida minayang kawigunan taleng sareng pepet. Pamargin paplajahan sane becik lan kaimbangin sareng tes pastika berpengaruh sareng pikolih panincapang kawagedan sisia ngwacen aksara Bali.

Pikolih sane kaping kalih inggih punika ngenenin indik panincapan kawagedan ngwacen aksara Bali sisia kelas IVA SD Negeri 2 Sakti Nusa Penida. Evaluasi anggen ngarereh kawagedan ngwacen aksara Bali inggih punika nganggen tes ngwacen sasuratan sane sampun kasiagayang olih guru. Tes punika kalaksanayang ring ungkur siklus. Ring tes ngwacen aksara Bali punika, sisia kaicen ngwacen sasuratan aksara Bali sane wenten ring topi. Tatujon sane kaaptiang penilik inggih punika sisia kaaptiang prasida ngwacen aksara Bali sane becik. Sisia taler uning aspek-aspek sane patut kauratiang ritatkala ngwacen aksara Bali mangda prasida nganutin aspek-aspek punika. 
Aspek sane katureksa anggen nentuang pikolih sisia ngwacen aksara Bali inggih punika aspek nguningin wentuk aksara Bali, ketepatan ngwacen kruna maaksara Bali, kejelasan ri kala ngwacen, lan keaktifan sisia. Ring aspek nguningin wentuk aksara Bali sisia mangda prasida uning indik wentuk lan bacaannyane. Ring aspek ketepatan mangda indik sasuratan lan sane kawacen mangda anut. Ring aspek kejelasan mangda sisia punika jelas lan tegas ri kala ngwacen. Lan ring aspek keaktifan sisia mangda prasida masaing sareng sawitrannyane.

Rerata pikolih sisia sadurung nganggen media paplajahan media topi aksara inggih punika 65,13 . Penilik ngamolihang pikolih ring siklus I punika prasida nincap dados 72,53. Ring siklus I puniki sane anut sareng KKM wantah $33,33 \% \quad(5$ sisia sane prasida ngamolihang nilai 75 utawi langkung). Risampune kalaksanayang siklus II, pikolihnyane prasida nincap dados 80,13 . Sisia sane prasida anut sareng KKM akehnyane $86,67 \%$ (13 sisia sane prasida molihang nilai 75 utawi langkung). Saking pikolih punika, prasida cutetang yening media paplajahan topi aksara puniki prasida kaanggen nincapang kawagedan ngwacen aksara Bali sisia kelas IVA SD Negeri 2 Sakti.

Panincapang puniki nenten lempas sareng kawigunan media topi aksara sane kaanggen ri tatkala ngamargiang paplajahan. Media topi aksara puniki prasida nincapang keaktifan sisia ring lelintihan pangajah-ajah, lan sisia nenten tegang ri tatkala nyarengin paplajahan.

Pikolih sane kaping tiga inggih punika ngenenin indik penampen sisia indik media peplajahan topi aksara sane kasurat nganggen aplikasi Balinese font and keyboard anggen nincapang kawagedan ngwacen aksara Bali sisia. Punika prasida kacihnayang saking siklus sane kapertama. Yening cingak ring pasinahan sane kapertama, kapolihang data makehnyane 9 sisia $(60,00 \%)$ ngicen panampen pinih cumpu, 5 sisia $(33,33 \%)$ ngicen panampen cumpu, lan 1 sisia $(6,67 \%)$ ngicen panampen kirang cumpu. Ring pasinahan kaping kalih kapolihang data penampen sisia, wenten 7 sisia $(46,67 \%)$ ngicen panampen pinih cumpu, 5 sisia $(33,33 \%)$ ngicen panampen cumpu, lan 3 sisia $(20,00 \%)$ ngicen panampen kirang cumpu. Pasinahan kaping tiga, kapolihang data makehnyane 10 sisia $(66,67 \%)$ ngicen panampen pinih cumpu, 4 sisia $(26,67 \%)$ ngicen panampen cumpu, lan wenten 1 sisia $(6,67 \%)$ ngicen panampen kirang cumpu. Pasinahan kaping pat, prasida kapolihang data makehnyane 7 sisia $(46,67 \%)$ ngicen panampen pinih cumpu, 6 sisia $(40,00 \%)$ ngicen panampen cumpu, lan 2 sisia $(13,33 \%)$ ngicen panampen kirang cumpu. Ring pasinahan kaping lima mikolihang panampen sisia sane pinih cumpu 13 sisia (86,67\%), 1 sisia $(6,67 \%)$ ngicen panampen cumpu, lan 1 sisia $(6,67 \%)$ mikolihang panampen kirang cumpu. Pasinahan kaping 6 prasida kapolihang dara wenten 9 sisia $(53,33 \%)$ sane ngicen panampen pinih cumpu, 4 sisia $(26,67 \%)$ ngicen panampen cumpu, lan 3 sisia $(20,00 \%)$ ngicen panampen kirang cumpu. Prasida kaacutetang ngenenin indik pikolih panampen sisia ring siklus I inggih punika saking 15 diri sisia sane nagingin angket utawi kuesioner, sane ngicen panampen positif pisan wenten 11 diri sisia lan sane ngicen panampen positif wenten 4 diri sisia. Skor rerata penampen sisia ngwacen aksara Bali antuk media topi aksara sane kasurat antuk Balinese font and keyboard inggih punika 26,87.

Yening pikolih panampen sisia ring siklus sane kaping kalih kasinahang ring pasinahan sane kapertama, kapolihang data makehnyane 10 sisia $(66,67 \%)$ ngicen panampen pinih cumpu, lan 5 sisia $(33,33 \%)$ ngicen panampen cumpu,. Ring pasinahan kaping kalih kapolihang data penampen sisia, wenten 7 sisia $(46,67 \%)$ ngicen panampen pinih cumpu, 5 sisia $(33,33 \%)$ ngicen panampen cumpu, lan 3 sisia $(20,00 \%)$ ngicen panampen kirang cumpu. Pasinahan kaping tiga, kapolihang data makehnyane 10 sisia $(66,67 \%)$ ngicen panampen pinih cumpu, 4 sisia $(26,67 \%)$ ngicen panampen cumpu, lan wenten 1 sisia $(6,67 \%)$ ngicen panampen kirang cumpu. Pasinahan kaping pat, prasida kapolihang data makehnyane 7 sisia $(46,67 \%)$ ngicen panampen pinih cumpu, 6 sisia $(40,00 \%)$ ngicen panampen cumpu, lan 2 sisia $(13,33 \%)$ ngicen panampen kirang cumpu. Ring pasinahan kaping lima mikolihang panampen sisia sane pinih cumpu 13 sisia $(86,67 \%), 1$ sisia $(6,67 \%)$ ngicen panampen cumpu, lan 1 sisia $(6,67 \%)$ mikolihang panampen kirang cumpu. Pasinahan kaping 6 prasida kapolihang dara wenten 10 sisia $(66,67 \%)$ sane ngicen panampen pinih cumpu, 3 sisia $(20,00 \%)$ ngicen panampen cumpu, lan 2 sisia $(13,33 \%)$ ngicen panampen kirang cumpu. Punika prasida kacihnayang saking angket puniki yening 13 sisia sane cumpu pisan $(86,67 \%)$, lan 2 sane cumpu $(13,33 \%)$. Prasida kacutetang ngnenin indik pikolih panampen sisia ring siklus II inggih punika saking 15 diri sisia sane nagingin angket utawi kuesioner, sane ngicen panampen positif pisan wenten 14 diri sisia lan sane ngicen panampen positif wenten 1 diri sisia. Skor rerata penampen sisia ngwacen aksara Bali antuk media topi aksara sane kasurat antuk Balinese font and keyboard inggih punika 27,20.

Prasida kacutetang, penampen sisia indik media pembelajaran topi aksara sane kasurat 
antuk aplikasi Balinese font and keyboard puniki ngranjing ring kategori positif pisan mawinan ring persentase punika akehan sisia sane ngrasayang seneng kaajahin nganggen media pembelajaran topi aksara punika. Data puniki kapolihang saking angket/kuesioner sane sampun kadagingin olih sisia.

Riantukan punika, saking angket punika taler penilik polih panampen yening sisia punika meled yening benjang pungkur guru punika nganggen media paplajahan sekadi penilik laksanayang mangda sisia punika nenten tegang lan marasa seneng ritatkala nyarengin paplajahan. Punika prasida kacingak saking pikolih angket ring siklus I lan II. Para sisia madue panampen indik aplikasi Balinese font and keyboard sane sasuratan aksaranyane becik lan rapi. Prasida kacingak ring lampiran indik kawentenan wentuk aksaranyane sane becik, lan aplikasi Balinese font and keyboard puniki mabinayan sareng aplikasi lianan santukan yening wenten surang sareng pepet punika nenten matumpuk, nika mawinan aplikasi puniki kabaos becik.

Prasida kacutetang, penampen sisia indik media pembelajaran topi aksara sane kasurat antuk aplikasi Balinese font and keyboard puniki ngranjing ring kategori positif pisan mawinan ring persentase punika akehan sisia sane ngrasayang seneng kaajahin nganggen media pembelajaran topi aksara punika.

Media pembelajaran topi aksara sane kaanggen ring paplajahan ngwacen aksara Bali prasida nincapang kawagedan ngwacen aksara sisia. Punika nyihnayang yening tetilikan puniki sampun tercapai. Riantukan punika, media pembelajaran topi aksara puniki sampun mabukti prasida kaanggen ring lelintihan pangajah-ajah anggen nincapang kawagedan ngwacen aksara Bali sisia kelas IVA SD Negeri 2 Sakti Nusa Penida.

Dadosnyane manut sareng sepat siku-siku sane sampun katelatarang punika, PTK puniki kalaksanayang antuk makudang-kudang siklus sane sistematis lan kaaptiang sumangdane PTK puniki prasida ngicenin paobahan, (Kunandar, 2009:43). Riantukan punika lelintihan pangajahajah ring sekolah punika nenten kabaos berhasil yening nenten kaimbangin sareng media pembelajaran, punika jakti prasida kadadosang perantara anggen ngicenin materi ajah, merangsang pikayun, minat lan panampen sisia punika mangda seneng mlajah. Punika pinaka pelaksana tetilikan sane sistematis sane prasida ngicenin paobahan pikolih sisia sane prasida ngamolihang panincapan kawagedan sisia ngwacen aksara Bali.

Kawagedan sisia punika prasida nincap mawinan kaimbangin antuk kaluihan media topi aksara sane kasurat antuk aplikasi Balinese font and keyboard. Wenten kaluihan media topi aksara minakadi 1) Kawentenan foto sisia sane ngawinang nenten tegang ri kala paplajahan ring kelas, 2) Media sane nenten mesuang biaya, mawinan ngrereh foto sisia usan punika ka edit ring HP, 3) Inih tekening prebea, artosnyane prasida kakaryanin ring makasamian genah, nenten kaiket antuk galah, (Effendi, 2005). Manut saking kaluwihan punika, dadosnyane paplajahan ngwacen aksara Bali nganggen media paplajahan topi aksara sane sampun kamargiang punika prasida kabaos becik lan menarik.

Wenten tatilik asoroh sane kacingakin panilik anggen sasuluhan minakadi pikolih sane kalaksanayang olih $\mathrm{Ni}$ Made Agnityas Prima Astuti sane mamurda "Pembelajaran Menulis Cerpen Dengan Menggunakan Media Komik Kelas XI Di SMA Negeri 2 Negara Tahun Ajaran 2011/2012". Ring tetilikan punika wenten panincapan nilai rerata 45,94 ring siklus I taler prasida nincap ring siklus II dados 78,5. Ring tetilikan punika prasida nyihnayang yening media komik punika prasida nincapang kawagedan nyurat cerpen sisia kelas XI SMA Negeri 2 Negara. Ring tetilikan puniki taler ngajahin sisia mangda rajin ngwacen buku. Taler tetilik sane kaping kalih saking I Made Dwi Adipraya (2015) sane mamurda "Iusan Pangawigunaan Piranti Gambar Monopoli Mabasa Bali Ring Paplajahan Ngwacen Aksara Bali Ring Sisia Kelas V SDK Marsudirini". Pikolih ring pratindakan punika 61,44, selanturnyane sesampune kalaksanayang siklus I punika prasida nincap 70,31 lan malih prasida nincap ring siklus II pikolihnyane 83,81. Pikolih sane kapolihang punika kabaos becik. Ring tetilik punika nganggen media gambar monopoli ritatkala ngalaksanayang paplajahan ring kelas.

\section{IMPLIKASI}

Sajeroning tetilikan puniki ring kawigunannyane dumogi prasida kaanggen uger-uger ri kala guru ngamargiang peplajahan mapaiketan ring peplajahan ngwacen aksara Bali. Sepatutnyane ngawigunayang media mangda manut dudonan tata cara sane patut anggen nagingin peplajahan ring kelas. Yening peplajahan indik ngwacen aksara Bali kasarengin media sane manut, sisia dangan pacang ngaresepang. Yening sampun ngresepang, sinah prasida nincapang kawagedan sisia, kaweruhan sisia, uratian sisia miwah sane tiosan. Prasida kacingak ring tetilikan puniki sampun mabukti kawagedan sisia nincap, inggih punika sampun prasida nincapang kawagedan sajeroning ngwacen aksara Bali nganggen media topi aksara sane kasurat antuk aplikasi Balinese font and 
keyboard saha sisia marasa seneng ri kala nyarengin paplajahan.

\section{PAMUPUT}

Malarapan antuk pikolih sane sampun katelatarang ring adiaya IV, prasida kacutetang ngenenin indik daging pikolih tetilik inggih punika : (a) Tetilik puniki klaksanayang antuk kalih siklus tur kalaksanayang antuk 4 patemon. Tetilikan puniki kalaksanayang ngawit pinanggal 17 April ngantos 17 Mei 2019. Tata titi sane pinih patut sajeroning nglaksanayang media topi aksara antuk aplikasi Balinese font and keyboard kaanggen nincapang kawagedan ngwacen aksara Bali sisia kelas IVA SD Negeri 2 Sakti Nusa Penida inggih punika: ring patemon kapertama (1) Guru ngicenin apersepsi sane mapaiketan sareng paplajahan sane sampun kamargiang awuku sane lintang, (2) Guru nelatarang daging paplajahan indik ngwacen aksara Bali, (3) Guru nelatarang indik artos, serana lan tata cara ngewacen aksara Bali antuk media topi aksara antuk aplikasi Balinese font and keyboard, (4) Guru ngicen galah sisia metaken, yening wenten sane durung karesepang, (5) Guru nyayagaang piranti sane kaanggen ring sajeroning ngamargiang paplajahan media topi aksara antuk aplikasi Balinese font and keyboard, (6) Guru nguningayang mangda sisia nyiagayang angga, (7) Guru ngicen siki sisia milih siki nomor tur ngwacen sasuratan sane wenten ring foto sisia sane nganggen topi, (8) Guru lan sisia sareng-sareng nureksain pakaryan sisia sane lianan, (9) Guru ngicen hadiah (penguatan) marupa emoticon sane prasida katempel ring baju, Ian sisia sane polih emoticon akeh sane polih jayanti tur polih nilai tambahan, (10) Guru lan sisia nyutetang paplajahan. Ring patemon kaping kalih (1) Guru ngicenin atur uning ngenennin indik paplajahan sane jagi kamargiang (nalatarang indikator lan tatujon paplajahan ngwacen aksara Bali), (2) Guru ngicenin apersepsi sane mapaiketan sareng paplajahan sane sampun kamargiang awuku sane lintang, (3) Guru nelatarang daging paplajahan indik ngwacen aksara Bali, (4) Guru ngicen galah sisia metaken, yening wenten sane durung karesepang, (5) Guru nyayagaang piranti sane kaanggen ring sajeroning test ngwacen aksara Bali, (6) Soang-soang sisia magentian ngwacen makudang-kudang lengkara maaksara Bali ring arep kelas, (7) Guru lan sisia nyutetang paplajahan. (b) Pangawigunan media topi aksara antuk aplikasi Balinese font and keyboard prasida kaanggen nincapang kawagedan ngwacen aksara Bali sisia kelas IVA SD Negeri 2 Sakti Nusa Penida. Indike punika prasida kacingakin sakin rata-rata pikolih sisia ngwacen aksara Bali. Rata-rata pikolih sisia sadurung nganggen edia topi aksara inggih punika 65,15. Ring siklus I sampun nincap dados 72,53 , ring siklus I puniki wau $5(33,33 \%)$ sisia sane prasida ngamolihang nilai langkung saking 75 . Nincap malih ring siklus II, rata-rata pikolih sisia inggih punika 80,13 . Presentase sisia sane sampun polih nilai langkung saking 75 inggih punika 13 (86,67\%) sisia. (c) Panampen sisia ring kawentenan media topi aksara antuk aplikasi Balinese font and keyboard puniki becik pisan. Sisia merasa seneng tur nenten jejeh yening paplajahan basa Bali kamargiang nganggen media sekadi punika. Indike punika prasida kacingakin saking angket penampen sisia sane sampun kadagingin olih sisia kelas IVA. Samian sisia ngicen panampen positif pisan ring kawentenan media topi aksara antuk aplikasi Balinese font and keyboard puniki. Malarapang antuk pikolih sane sampun wenten sajeroning tetilikan puniki, salanturnyane prasida kamahbahang piteket sekadi (1) Guru basa Bali ring SD Negeri 2 Sakti Nusa Penida mangda prasida ngawentenang pangajahan sane variatif lan inovatif sane ngawinang sisia sayan seneng nyarengin pangajahan basa Bali. Media topi aksara antuk aplikasi Balinese font and keyboard puniki pastika prasida ngawinang panincapan nilai sisia miwah ngawinang sisia sayan seneng nyarengin paplajahan ngwacen aksara Bali. Kawentenan foto sawitrannyane ring pamargin paplajahan nase ngawinang sisia sayan seneng ri kala mlajah. Santukan sisia kelas IV seneng yening wenten plalianan ring paplajahan. (2) Sisia SD Negeri 2 Sakti Nusa Penida mangda setata eling ring napi sane kaplajahin, santukan samian pangajahan sane kamargiang tetujonnyane mangda sisia sayan waged. Samian sisia mangda prasida ngresep ring paplajahan sane kamargiang mangda prasida sayan waged. Tetujon saking pengajahan punika nenten lianan anggen ngelimbakang pangajahan sane kapolihang. (3) Calon guru basa Bali prasida makarya media paplajahan menarik, taler prasida kawewehin malih antuk metode sane lianan. Ring tetilikan puniki sampun kacingak pikolih sane becik, pastika media sane lianan prasida malih sayan becik kamargiang ring sajeroning ngamargiang profesi dados guru basa Bali. (4) Majeng ring panilik tiosan kaptiang prasida nglaksanayang tetilikan malih ngeninin indik media paplajahan antuk kawagedan sane lianan, punika prasida ngawinang pangajahan ngeninin indik media puniki sayan variatif lan inovatif miwah prasida malih kadadosang silih sinunggil pangajahan sane ngawentenang inspirasi pangwacen tetilikan. 
Malarapan antuk pikolih sane sampun katelatarang ring adiaya IV, prasida kacutetang ngenenin indik daging pikolih tetilik puniki, sekadi: tetilik puniki klaksanayang antuk kalih siklus tur kalaksanayang antuk 4 patemon. Tetilikan puniki kalaksanayang ngawit pinanggal 17 April ngantos 17 Mei 2019. Pangawigunan media topi aksara antuk aplikasi Balinese font and keyboard prasida kaanggen nincapang kawagedan ngwacen aksara Bali sisia kelas IVA SD Negeri 2 Sakti Nusa Penida. Indike punika prasida kacingakin sakin rata-rata pikolih sisia ngwacen aksara Bali. Rata-rata pikolih sisia sadurung nganggen edia topi aksara inggih punika 65,15. Ring siklus I sampun nincap dados 72,53, ring siklus I puniki wau 5 (33,33\%) sisia sane prasida ngamolihang nilai langkung saking 75. Nincap malih ring siklus II, rata-rata pikolih sisia inggih punika 80,13. Presentase sisia sane sampun polih nilai langkung saking 75 inggih punika $13(86,67 \%)$ sisia. Panampen sisia ring kawentenan media topi aksara antuk aplikasi Balinese font and keyboard puniki becik pisan. Sisia merasa seneng tur nenten jejeh yening paplajahan basa Bali kamargiang nganggen media sekadi punika. Indike punika prasida kacingakin saking angket penampen sisia sane sampun kadagingin olih sisia kelas IVA. Lianan sareng tetingkesan punika, pacang katelatarang piteketnyane, minakadi: 1) majeng ring sisia SD Negeri 2 Sakti Nusa Penida mangda setata eling ring napi sane kaplajahin, santukan samian pangajahan sane kamargiang tetujonnyane mangda sisia sayan waged. 2) majeng ring guru basa Bali ring SD Negeri 2 Sakti Nusa Penida mangda prasida ngawentenang pangajahan sane variatif lan inovatif sane ngawinang sisia sayan seneng nyarengin pangajahan basa Bali. 3) majeng ring calon guru basa Bali prasida makarya media paplajahan menarik, taler prasida kawewehin malih antuk metode sane lianan. 4). majeng ring panilik tiosan kaptiang prasida nglaksanayang tetilikan malih ngeninin indik media paplajahan antuk kawagedan sane lianan, punika prasida ngawinang pangajahan ngeninin indik media puniki sayan variatif lan inovatif miwah prasida malih kadadosang silih sinunggil pangajahan sane ngawentenang inspirasi pangwacen tetilikan.

\section{KEPUSTAKAAN}

Arikunto, 2006. Dasar-dasar Evaluasi Pendidikan. Jakarta:PT.Bumi Aksa.

Efendi , Dr. M.Pd. 2011. Pembelajaran Berbasis Teknologi Informasi dan Komunikasi. Jakarta: Rajawali Pers.

Sanjaya, H. Wina. 2012. Perencanaan dan Desain Pembelajaran. Jakarta: Fajar Interpratama Offset.

Sutama, I Made. 2009. Pedoman Penulisan Skripsi dan Tugas Akhir. Singaraja: Undiksha.

Suwija, I Nyoman. 2014. Tata Titi Mabaos Bali. Denpasar: Pelawasari.

Wendra, I Wayan. 2012. Buku Ajar Penulisan Karya Ilmiah. Singaraja: Undiksha

Surya, Dr. M.Pd. 2012. Pengembangan Media dan Sumber Pembelajaran. Jakarta: Prestasi Pustaka 